\title{
Effect of Early Nutrition Intervention on Advanced Nasopharyngeal Carcinoma Patients Receiving Chemoradiotherapy
}

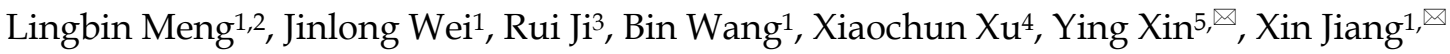 \\ 1. Department of Radiation Oncology, the First Hospital of Jilin University, Changchun, China \\ 2. Department of Internal Medicine, Florida Hospital, Orlando, FL 32804, USA \\ 3. Department of Biology, Valencia College, Orlando, FL 32825, USA \\ 4. Department of Clinical Cancer Prevention, The University of Texas M. D. Anderson Cancer Center, Houston, TX 77030, USA \\ 5. The Key Laboratory of Pathobiology, Ministry of Education, Jilin University, Changchun, China
}

$\square$ Corresponding authors: Prof. Ying Xin, Ph.D, The Key Laboratory of Pathobiology, Ministry of Education, Jilin University, 126 Xinmin Street, Changchun, 130021 China, email: xiny@jlu.edu.cn; Prof. Xin Jiang, Ph.D, Department of Radiation Oncology, The First Hospital of Jilin University, 71 Xinmin Street, Changchun 130021China, Email: rji@mail.valenciacollege.edu

(c) Ivyspring International Publisher. This is an open access article distributed under the terms of the Creative Commons Attribution (CC BY-NC) license (https://creativecommons.org/licenses/by-nc/4.0/). See http://ivyspring.com/terms for full terms and conditions.

Received: 2019.01.24; Accepted: 2019.05.11; Published: 2019.06.09

\begin{abstract}
Background: Patients with nasopharyngeal carcinoma (NPC) frequently developed the problem of malnutrition at the time of diagnosis. Chemoradiotherapy (CRT) can even worsen the situation. Therefore, nutritional intervention should be applied to prevent CRT-associated weight loss and interruption of CRT. However, it is still controversial if early nutritional intervention is beneficial to NPC patients with CRT. This study is to investigate the influence of early nutritional intervention on advanced NPC patients with CRT by evaluating the nutritional status and CRT treatment tolerance.

Methods: A cohort of 78 stage III-IV nasopharyngeal carcinoma patients was divided into early $(n=46)$ and late $(n=32)$ nutrition intervention groups. The early group of patients received nutritional support at the beginning of CRT, whereas the late group received such a support until development of the side effects, like $50 \%$ required oral dietary intake or $>10 \%$ weight loss. The data were collected and statistically analyzed.

Results: There was no significant difference in baseline clinical characteristics between these two groups, suggesting that no selection bias occurred. Both groups of patients had weight loss at the end of CRT and 3 months thereafter. However, at the later time point, the early group started to regain their weight, while the late group continued to lose weight. At both time points, the early group had a lower percentage of weight loss than the late group. Similar results were also obtained for BMI, albumin, and pre-albumin levels (All $p<0.05$ ). Besides, the early group showed a lower rate of advanced mucositis, a lower percentage of patients with more than 3 days RT breaks, fewer days of RT delayed for toxicity, and a lower percentage of patients with unplanned hospitalizations (All $p<0.05$ ). A linear correlation was also found between the percentage of weight loss and the number of days of RT delayed.

Conclusion: Early nutritional intervention provides beneficial outcomes to NPC patients by maintaining their nutritional status and enhancing CRT treatment tolerance. Our results also indicated early nutrition intervention may reduce the hospital cost and improve patients' life quality.
\end{abstract}

Key words: nasopharyngeal carcinoma, chemoradiotherapy, nutritional intervention, advanced mucositis

\section{Introduction}

Nasopharyngeal carcinoma (NPC), the most common cancer originating in the nasopharynx area, is a squamous cell carcinoma or non-keratinizing, undifferentiated type of carcinoma $[1,2]$. It is a significant health problem in the world, especially in the Southern China and Southeast Asia [3, 4]. The NPC staging system was developed by the American Joint Committee on Cancer (AJCC) and the 
International Union Against Cancer (UICC) based on tumor stage, lymph node involvement, and distant metastasis [5, 6]. For the advanced NPC (Stage III/IV), the U.S. National Comprehensive Cancer Network (NCCN) Guidelines recommend CRT adjuvantly and neoadjuvantly as the standard treatment $[7,8]$. Recently, the therapeutic advancement, e.g., the intensity-modulated radiotherapy (IMRT), significantly improved survival of patients [9-11]. With CRT treatment, approximately $80 \%$ of NPC patents can survive for at least 5 years [12-14].

Malnutrition is an important clinical factor in cancer progression and treatment. It results from a state when food intake fails to provide the energy requirements [15]. Malnutrition is very common in NPC patients because the original tumor site could significantly reduce food intake [16, 17]. Also, the majority of NPC patients have unhealthy habits that worsen the malnutrition, like tobacco smoking and alcohol consumption [18]. Furthermore, CRT frequently exacerbates malnutrition because of its adverse effects, such as alteration of taste, advanced mucositis, fatigue, nausea, vomiting, and so on [19, 20]. Among the CRT side effects, advanced mucositis (Stage III/IV) is the most common cause of severe malnutrition and sometimes, the worsened malnutrition may even disrupt CRT durations [21, 22]. Therefore, in order not to compromise CRT treatment efficacy, nutrition intervention should be applied to maintain the nutritional status of NPC patients with CRT.

International guidelines suggest intensive nutritional counseling (NC) and oral nutritional supplements as nutritional intervention for head and neck cancer patients with CRT [23-25]. It is also suggested that if the cancer affects eating or swallowing, enteral nutrition (EN) should be provided through tube feeding [26]. The delivery of tube feeding can either be nasogastric tubing (NG) or percutaneous gastrostomy (PEG). Following these guidelines, a study showed that an early nutritional intervention could efficiently reduce weight loss, interruption of radiation treatment, and unplanned hospitalizations in head and neck cancer patients with CRT [27]. Thereafter, few studies have been conducted to evaluate the effect of the early nutrition intervention on NPC patients with CRT. A recent study reported that the nutritional intervention failed to decrease the declination of body weight, BMI serum albumin, and pre-albumin levels or the prognostic nutritional index in advanced NPC patients with CRT [28]. However, this study had a significant selection bias and a small patient number, and thus the result could be considered as unreliable.
In this study, we investigated the effect of the early nutritional intervention on advanced NPC patients with CRT by evaluating the nutritional status and CRT treatment tolerance. Clinical factors, including body weight, BMI, serum albumin, and pre-albumin, were collected to observe the nutritional status. Data on the incidence of advanced mucositis, rate of patients with RT interruptions, days of RT delayed, and rate of patients with unplanned hospitalizations were calculated to assess the treatment tolerance.

\section{Patients and Methods}

\section{Study subjects}

This study included 78 NPC patients who received medical treatments between March 2015 and March 2018 in The First Hospital of Jilin University (Jilin, China). The inclusion criteria were 1). Patients were histopathologically diagnosed with III/IV NPC; 2). Patients were aged between 18 and 70 years old; 3 ). Patients had no significant liver and kidney diseases, heart and lung dysfunctions, or serious endocrine and metabolic diseases to receive initial chemoradiotherapy; and 4). Patients had a Karnofsky Performance Status (KPS) score of 70 or above. This study was approved by the Ethics Committee of The First Hospital of Jilin University and waived for individual informed consent.

\section{Chemoradiotherapy}

All patients were treated with IMRT delivered as five fractions per week. All patients were immobilized in the supine position with a head, neck, and shoulder thermoplastic mask. All patients were scanned with serial 3-mm slices from the vertex through the clavicles. The PTV was constructed automatically based on each volume with an additional 3-mm margin in three dimensions to account for set-up variability. The prescribed radiation dose was 69-74 Gy to PGTVnx, 66-70 Gy to PGTVnd, 60-66 Gy to PTV1, and 50-54 Gy to PTV2, delivered in 30 or 33 fractions. Radiation was delivered once daily, five fractions per week, over 6-6.5weeks for IMRT planning. The dose to OAR was limited on the basis of the RTOG 0225 protocol.

Neoadjuvant chemotherapy consisted of TPF (docetaxel $60 \mathrm{mg} / \mathrm{m}^{2} /$ day on day 1 , cisplatin 25 $\mathrm{mg} / \mathrm{m}^{2} /$ day on days 1 to 3 , and 5 -fluorouracil 500 $\mathrm{mg} / \mathrm{m}^{2} /$ day on days 1 to 3 ), TP (docetaxel 75 $\mathrm{mg} / \mathrm{m}^{2} /$ day on day 1 , cisplatin $25 \mathrm{mg} / \mathrm{m}^{2} /$ day on days 1 to 3 ) and FP (cisplatin $25 \mathrm{mg} / \mathrm{m}^{2} /$ day on days 1 to 3 , and 5 -fluorouracil $500 \mathrm{mg} / \mathrm{m}^{2} /$ day on days 1 to 3 ) and every 3 weeks for 2-3 cycles. In concurrent chemotherapy, patients received a $30 \mathrm{mg} / \mathrm{m} 2 /$ week cisplatin or 80 to $100 \mathrm{mg} / \mathrm{m} 2 / \mathrm{q} 3 \mathrm{w}$ cisplatin regimen. 


\section{Nutrition Intervention}

These patients were divided into two groups, i.e., the early $(n=46)$ and late $(n=32)$ nutritional intervention groups. The early group of patients received nutritional support (see below for details) at the beginning of CRT (T0), while the late group didn't receive such a support until the side effects were developed, like $50 \%$ required oral dietary intake or $>10 \%$ weight loss. Both groups of patients continuously received such a nutritional support until 3 months after CRT.

The nutritional intervention was initially administered by oral nutrition (a commercial product) and if oral intake was not sufficient to maintain patients' nutritional status, short-term parental nutrition was applied. When patients formed severe oral mucositis causing dysphagia, enteral tube feeding (NGT or PEG) may be utilized.

\section{Data Collection}

Clinicopathological data were retrospectively collected from their medical records, like age, sex, weight, BMI, serum albumin and pre-albumin levels, history of tobacco smoking and alcohol consumption, incidence of III/IV mucositis and total radiotherapy dose. The nutritional status, including the body weight, BMI, and serum albumin and pre-albumin, was evaluated in both groups at the end of CRT (T1) and 3 months (T2) after chemoradiotherapy. To evaluate the treatment tolerance, we assessed the incidence of advanced mucositis, radiotherapy interruptions or delay, and unplanned hospitalization during the T1. For hospitalization criteria, patients will be admitted to hospital if the following conditions are presented: duration of III or IV mucositis is more than one week; duration of stage II fatigue is more than one week; weight loss is more than $10 \%$; stage III bone marrow suppression is developed with a fever.

\section{Statistical Analysis}

The data were statistically analyzed by using SPSS software (version 22.0; IBM SPSS, Chicago, IL, USA). The distributions of patients' characteristics between the groups were assessed by using Student's t-test for continuous variables and chi-square test or Fisher's exact test for categorical variables. A $P<0.05$ was considered as statistically significant.

\section{Results}

\section{Patient characteristics}

In this cohort of patients, there were 59 males and 19 females with a mean age of 51 years old. $68 \%$ smoked tobacco and $27 \%$ consumed excessive alcohol.
Staging data showed 23 patients at stage T1-2, 55 at T3-4, 66 at N0-2, and 12 at N3. Laboratory data showed a mean of $68 \mathrm{~kg}$ body weight, $41.8 \mathrm{~g} / \mathrm{L}$ of serum albumin level. All of these patients received CRT. As shown in Table 1, there was no difference in these clinicopathological factors between the early $(n=46)$ and late $(n=32)$ groups of patients $(p>0.05)$, indicating that there was no selection bias in these patients. However, there was a difference in the incidence of III/IV mucositis $(p<0.05)$. The lower ratio of advanced mucositis in the late group probably explained why this group of patients refused nutrition intervention at the beginning of CRT.

Table 1. Baseline characteristics of early nutrition intervention group and late nutrition intervention group.

\begin{tabular}{|c|c|c|c|c|}
\hline \multicolumn{2}{|l|}{ Characteristics } & Early Group (46) & Late Group (32) & $p$ value \\
\hline \multicolumn{2}{|l|}{ Age (years) } & $52.0 \pm 8.5$ & $49.5 \pm 9.2$ & 0.268 \\
\hline \multicolumn{2}{|l|}{ Sex ratio $(\mathrm{M} / \mathrm{F})$} & $34 / 12$ & $25 / 7$ & 0.345 \\
\hline \multicolumn{2}{|l|}{ Weight $(\mathrm{kg})$} & $67.5 \pm 9.8$ & $69.0 \pm 10.5$ & 0.629 \\
\hline \multicolumn{2}{|c|}{ Body mass index $(\mathrm{kg} / \mathrm{m} 2)$} & $24.9 \pm 3.2$ & $25.4 \pm 4.0$ & 0.598 \\
\hline \multicolumn{2}{|c|}{ Weight loss (\%) in the last 6 months } & $2.9 \pm 4.1$ & $2.4 \pm 3.3$ & 0.228 \\
\hline \multicolumn{2}{|c|}{ Albumin $(\mathrm{g} / \mathrm{L})$} & $41.6 \pm 3.3$ & $42.0 \pm 3.8$ & 0.526 \\
\hline \multicolumn{2}{|c|}{ Pre-albumin (mg/L) } & $265.4 \pm 49.3$ & $272.9 \pm 54.1$ & 0.187 \\
\hline \multicolumn{2}{|c|}{ Lymphocyte (/ul) } & $1760 \pm 512$ & $1820 \pm 715$ & 0.868 \\
\hline \multicolumn{2}{|c|}{ Cholesterol (mg/dl) } & $182 \pm 42$ & $188 \pm 57$ & 0.678 \\
\hline \multicolumn{2}{|c|}{ III, IV mucositis, n (\%) } & $6(10.87)$ & $1(3.13)$ & 0.032 \\
\hline \multirow[t]{2}{*}{ T stage, n (\%) } & $\mathrm{T} 1-2$ & $15(32.60)$ & $8(25)$ & 0.126 \\
\hline & T3-4 & $31(67.39)$ & $24(75)$ & \\
\hline \multirow[t]{2}{*}{ N stage, n (\%) } & N0-2 & $40(86.96)$ & $26(81.25)$ & 0.257 \\
\hline & N3 & $6(13.04)$ & $6(18.75)$ & \\
\hline \multicolumn{2}{|c|}{ Total dose of planned RT (Gy) } & $66.5 \pm 2.4$ & $67.0 \pm 2.6$ & 0.408 \\
\hline
\end{tabular}

\section{Better nutritional status in early nutrition intervention group}

To assess the nutritional status of patients, we recorded changes in body weight, BMI, serum albumin, pre-albumin, and total lymphocyte count, total cholesterol levels in both groups at the end of CRT (T1) and 3 months thereafter (T2). Figure 1A showed that both groups had weight loss at T1, while the early group started to regain the body weight at $\mathrm{T} 2$, whereas the late group continued to lose the body weight at T2. Furthermore, the early group had a lower frequency of weight loss than the late group at both T1 and T2 (Figure 2A). Similar results were also shown for the BMI, serum albumin and pre-albumin levels (Figure 1 and 2B-D, $p<0.05$ ). For total lymphocyte count and cholesterol level, the change pattern is similar but no significant difference was observed between the two groups (Supplemental figure).

\section{Increased CRT tolerance in early nutrition intervention group}

As shown in Table 2, the early group had a lower rate of advanced mucositis, therapy discontinuation or delay, and unplanned hospitalization at the end of 
CRT. Moreover, there was a significant linear association of weight loss and the number of days of RT delay in both study populations $(\mathrm{r}=-0.42 ; p<0.01)$. These results demonstrated that the early nutrition intervention was able to increase CRT tolerance possibly through maintenance of nutritional status.

Table 2. Comparing incidence of III\&IV mucositis, rate of patients with CRT interruptions, days of CRT delayed for toxicity, and rate of patients with unplanned hospitalizations between the early and late group at T1.

\begin{tabular}{llll}
\hline & Early & Late & $p$ \\
\hline III\&IV mucositis (\%) & 13.0 & 21.9 & 0.028 \\
Patients who had CRT breaks (>3 days) (\%) & 10.9 & 25.0 & 0.017 \\
Days of CRT delayed for toxicity & $2.2 \pm 1.8$ & $3.1 \pm 3.2$ & 0.033 \\
Rate of patients with unplanned hospitalizations & 13.0 & 31.3 & 0.009 \\
\hline
\end{tabular}

\section{Adverse events}

We then compare other adverse events of the CRT between these two groups of patients. However, unlike advanced mucositis, there was no significant difference found in hematology parameters, dermatitis, nausea, vomiting, xerostomia, nephrotoxicity or neurotoxicity (All $p>0.05$ ).

\section{Discussion}

Nutrition intervention has been demonstrated to play an important role in maintenance of the nutritional status and increasing CRT treatment

A

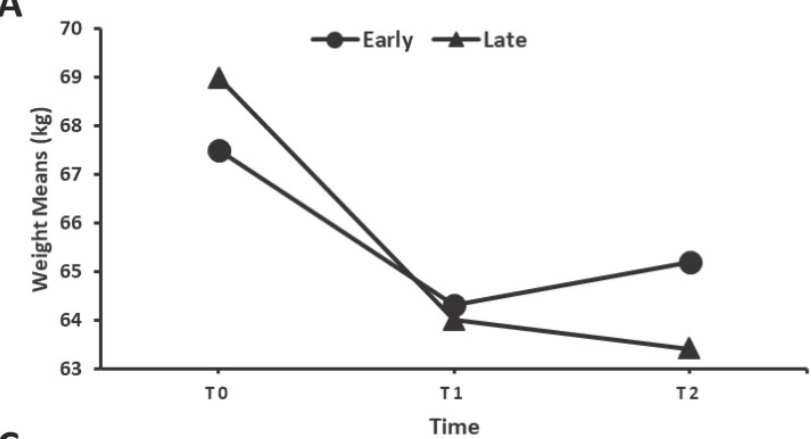

C

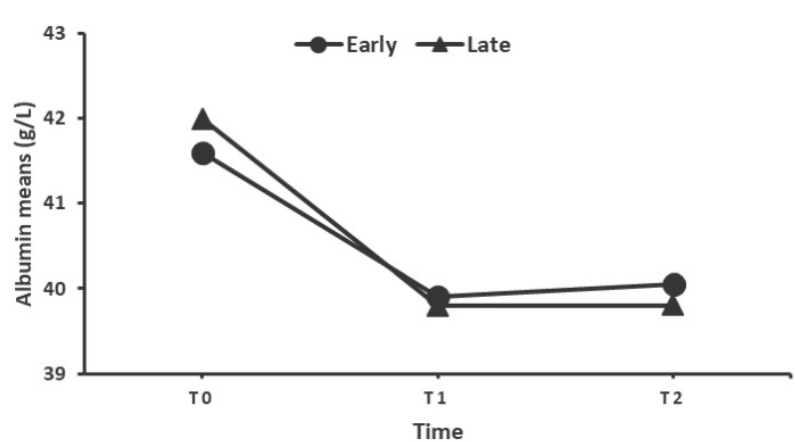

tolerance in head and neck patients [27]. Nevertheless, it is still controversial whether the nutrition intervention is beneficial to NPC patients with CRT [28]. A recent study showed nutritional intervention had no improvement on maintenance of body weight, BMI, serum albumin, and pre-albumin levels [28]. In the present study, we showed that the early nutrition intervention significantly reduced declination of body weight, BMI, serum albumin and pre-albumin levels. Moreover, our results revealed that the early nutrition intervention decreased incidence of advanced mucositis and increased CRT treatment tolerance. Such a benefit could be possibly derived from maintenance of nutritional status.

Indeed, our current data are consistent with previous studies, further supporting importance of the nutrition intervention [19, 27, 29]. It has been frequently reported that patients would lose $10 \%$ or higher of body weight loss after CRT [30-32]. In the late group of patients in our current study, patients only lost $7.2 \%$ of their baseline weight, suggesting the nutrition intervention had the beneficial effect. More importantly, the early group had a significantly lower percentage of changes in body weight, BMI, serum albumin and pre-albumin, indicating that the early nutrition intervention was even more beneficial. Based on this finding, we speculated that an earlier nutrition support could even provide better CRT outcomes.
B

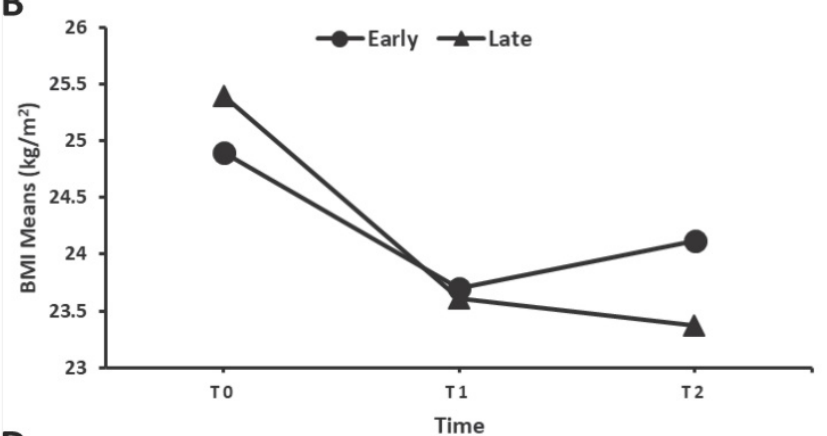

D

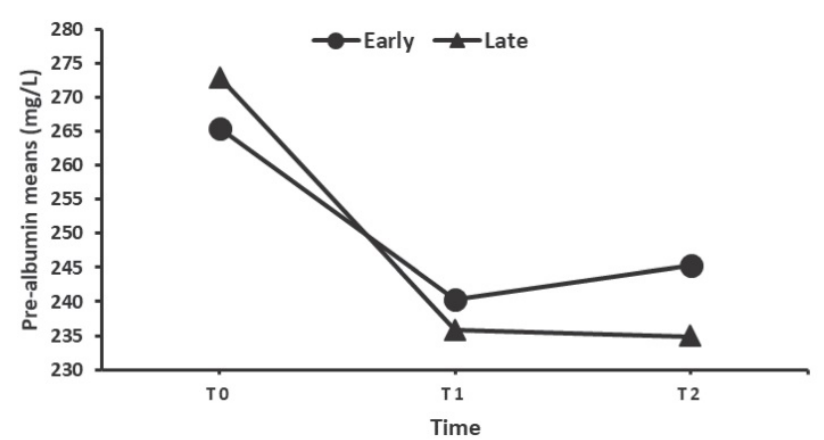

Figure 1. Means of weight (A), BMI (B), Albumin (C), and Pre-albumin levels (D) in the early and late groups over CRT treatment up to 3 months after the end of treatment. (T0: beginning of CRT, T1: end of CRT, T2: 3 months after CRT.) 
A

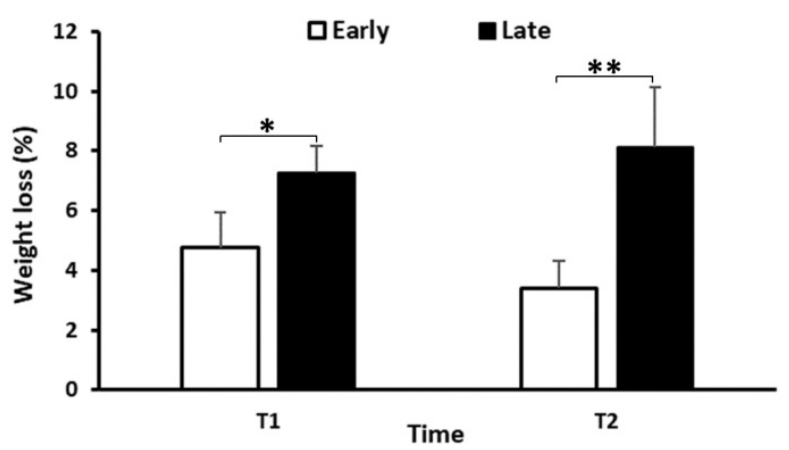

C

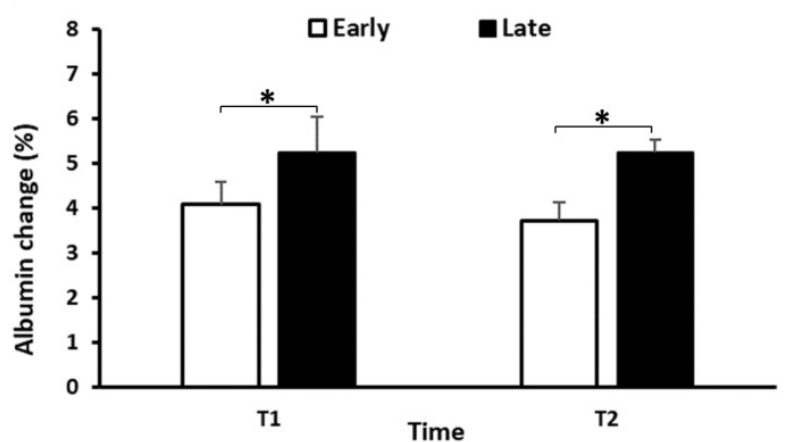

B

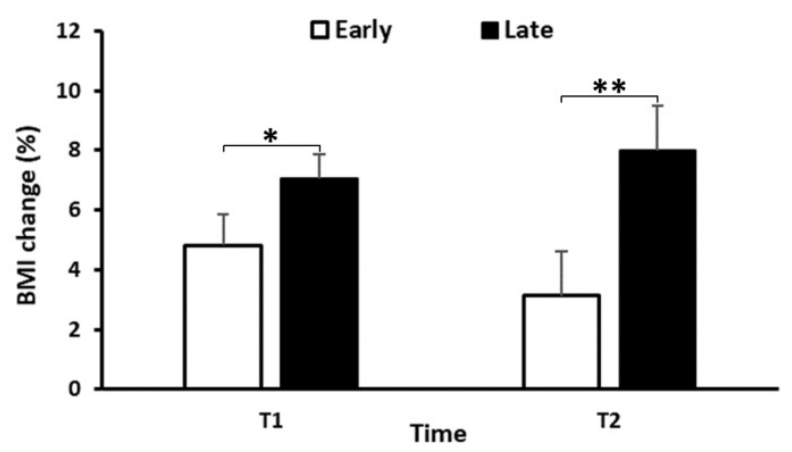

D

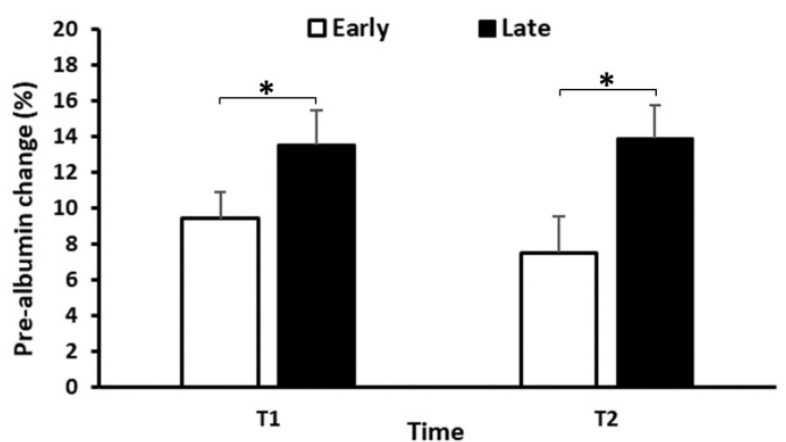

Figure 2. Percentage of weight loss (A), BMI change (B), Albumin change (C), and Pre-albumin change (D) from the beginning of CRT in the early and late groups. Data are represented as a mean \pm SD. $* p<0.05, * * p<0.01$.

However, a recent study didn't advocate the role of the nutrition intervention on NPC patients with CRT [28]. In that study, there was no significant difference in body weight change observed between nutrition intervention and control groups. Actually, that study showed limitations, e.g., their nutrition intervention protocol only included oral nutrition and parental nutrition supports, but there were no NG or PEG interventions. The two tube feedings are highly recommended as enteral nutritional support methods, especially when severe local mucositis developed [33]. It was demonstrated that NG or PEG was more effective to maintain nutritional status compared to oral nutrition alone [34, 35]. Moreover, more importantly, there was a selection bias between the nutrition and control groups in that study (28). BMI in the control group was significantly higher at the beginning of CRT, indicating that a better nutritional status in the control group might have protected patients from CRT-induced weight loss and thus caused a false null conclusion about nutrition intervention.

It is also worth mentioning that our current study did have a selection bias as well, i.e., the rate of advanced mucositis was significantly higher in the early group, possibly explaining why this group of patients accepted the early nutrition intervention. Nevertheless, the early group with initially higher rate of advanced mucositis, successfully reversed the result. At the end of CRT, the early group of patients showed a dramatically lower incidence of advanced mucositis compared to that of the late group $(13.0 \%$ vs $21.9 \%, \mathrm{p}<0.05)$, implying that the early nutrition intervention benefited patients' oral condition. Since severe mucositis is the most common CRT side effects and could result in CRT interruptions, we compared the rate of patients with RT interruptions, days of RT delayed, and rate of patients with unplanned hospitalizations at the end of CRT. Our results showed that the early group had a lower percentage of more than 3 days RT breaks, fewer days of RT delayed, and unplanned hospitalizations, suggesting that the early nutrition intervention increased CRT tolerance. We also found a significant linear correlation between percentage of weight loss and days of RT delayed, indicating that the early nutrition intervention increased CRT tolerance possibly through maintenance of the nutritional status. However, for other adverse effects than mucositis, we didn't observe any significant difference as shown in Table 3.

CRT interruption is an important clinical factor as it is a detrimental factor of NPC prognosis [36-38]. Therefore, the early nutrition intervention may provide better survival benefits to NPC patients. A future study with survival data of patients could help us to evaluate such a benefit of the early nutrition intervention in order to support this speculation. 
Also, the rate of unplanned hospitalizations is closely associated with hospital costs and quality of patients' life [39]. Our current results indicated that the early nutrition intervention could reduce the cost and improve the quality of life. However, as limited data access, we couldn't provide this evaluation.

Table 3. Adverse events at the end of CRT. ns: not significant.

\begin{tabular}{|c|c|c|c|c|}
\hline Adverse events & & Early Group (46) & Late Group (32) & $p$ value \\
\hline \multirow{4}{*}{$\begin{array}{l}\text { Hematological } \\
\mathrm{n}(\%)\end{array}$} & Anemia & $1(2.2)$ & $0(0)$ & ns \\
\hline & Thrombocytopenia & $3(6.5)$ & $2(6.3)$ & 0.77 \\
\hline & Neutropenia & $6(13.0)$ & $4(12.5)$ & 0.68 \\
\hline & Leucopenia & $7(15.2)$ & $4(12.5)$ & 0.55 \\
\hline \multirow{6}{*}{$\begin{array}{l}\text { Non-hematological } \\
\mathrm{n}(\%)\end{array}$} & Dermatitis & $6(13.0)$ & $5(15.6)$ & 0.48 \\
\hline & Nausea & $10(21.7)$ & $6(18.8)$ & 0.26 \\
\hline & Vomiting & $5(10.9)$ & $3(9.3)$ & 0.65 \\
\hline & Xerostomia & $2(4.3)$ & $1(3.1)$ & 0.55 \\
\hline & Nephrotoxicity & $0(0)$ & $0(0)$ & ns \\
\hline & Neurotoxicity & $1(2.2)$ & $0(0)$ & ns \\
\hline
\end{tabular}

Our study does have several limitations: it is a retrospective study and the sample size is relatively small. In the future, we will perform a prospective investigation of early nutritional intervention for different cancer patients during chemoradiotherapy to verify our current data.

In conclusion, the early nutrition intervention benefits NPC patients by maintaining nutritional status and improving CRT treatment tolerance, suggesting that NPC patients should receive the nutrition intervention as early as possible to get clinical benefits.

\section{Supplementary Material}

Supplementary figure.

http://www.jcancer.org/v10p3650s1.pdf

\section{Acknowledgements}

The authors would like to thank Xuebo Yan of The UT M. D. Anderson Cancer Center (Houston, TX, USA) for technical assistance.

This work was supported in part by grants from the National Natural Science Foundation of China (81570344, to Ying Xin; 81670353 to Xia Yin), the Norman Bethune Program of Jilin University (2015225, to Ying Xin and 2015203, to Xin Jiang), the Jilin Provincial Science and Technology Foundations (20180414039GH to Ying Xin and 20190201200JC to Xin Jiang).

\section{Author's contributions}

$\mathrm{LM}, \mathrm{YX}$, and $\mathrm{XJ}$ designed the project and wrote the paper. LM, JW, RJ, and BW performed experiments and generated data. XX reviewed and edited the manuscript. All authors approved the final manuscript.

\section{Competing Interests}

The authors have declared that no competing interest exists.

\section{References}

1. Mimi CY, Yuan J-M. Epidemiology of nasopharyngeal carcinoma. Seminars in cancer biology: Elsevier; 2002. p. 421-9.

2. Vokes EE, Liebowitz DN, Weichselbaum RR. Nasopharyngeal carcinoma. The Lancet. 1997; 350: 1087-91.

3. Jia W-H, Huang Q-H, Liao J, Ye W, Shugart $Y$, Liu Q, et al. Trends in incidence and mortality of nasopharyngeal carcinoma over a 20-25 year period (1978/1983-2002) in Sihui and Cangwu counties in southern China. BMC cancer. 2006; 6: 178.

4. Mutirangura A, Pornthanakasem W, Theamboonlers A, Sriuranpong V, Lertsanguansinchi P, Yenrudi S, et al. Epstein-Barr viral DNA in serum of patients with nasopharyngeal carcinoma. Clinical Cancer Research. 1998; 4: 665-9.

5. Kamran SC, Riaz N, Lee N. Nasopharyngeal carcinoma. Surgical Oncology Clinics. 2015; 24: 547-61.

6. Au JS, Law C, Foo W, Lau W. In-depth evaluation of the AJCC/UICC 1997 staging system of nasopharyngeal carcinoma: prognostic homogeneity and proposed refinements. International Journal of Radiation Oncology* Biology* Physics. 2003; 56: 413-26.

7. Chan A, Teo P, Ngan R, Leung T, Lau W, Zee B, et al. Concurrent chemotherapy-radiotherapy compared with radiotherapy alone in locoregionally advanced nasopharyngeal carcinoma: progression-free survival analysis of a phase III randomized trial. Journal of clinical oncology. 2002; 20: 2038-44.

8. Chua ML, Wee JT, Hui EP, Chan AT. Nasopharyngeal carcinoma. The Lancet. 2016; 387: 1012-24

9. Lee N, Xia P, Quivey JM, Sultanem K, Poon I, Akazawa C, et al. Intensity-modulated radiotherapy in the treatment of nasopharyngeal carcinoma: an update of the UCSF experience. International Journal of Radiation Oncology* Biology* Physics. 2002; 53: 12-22.

10. Pow EH, Kwong DL, McMillan AS, Wong MC, Sham JS, Leung LH, et al. Xerostomia and quality of life after intensity-modulated radiotherapy vs. conventional radiotherapy for early-stage nasopharyngeal carcinoma: initial report on a randomized controlled clinical trial. International Journal of Radiation Oncology* Biology* Physics. 2006; 66: 981-91.

11. Li A-C, Xiao W-W, Shen G-Z, Wang L, Xu A-A, Cao Y-Q, et al. Distant metastasis risk and patterns of nasopharyngeal carcinoma in the era of IMRT: long-term results and benefits of chemotherapy. Oncotarget. 2015; 6: 24511.

12. Peng G, Wang T, Yang K-y, Zhang S, Zhang T, Li Q, et al. A prospective, randomized study comparing outcomes and toxicities of intensity-modulated radiotherapy vs. conventional two-dimensional radiotherapy for the treatment of nasopharyngeal carcinoma. Radiotherapy and oncology. 2012; 104: 286-93.

13. Lai S-Z, Li W-F, Chen L, Luo W, Chen Y-Y, Liu L-Z, et al. How does intensity-modulated radiotherapy versus conventional two-dimensional radiotherapy influence the treatment results in nasopharyngeal carcinoma patients? International Journal of Radiation Oncology* Biology* Physics. 2011; 80: 661-8.

14. Lee AW, Ng WT, Chan LL, Hung WM, Chan CC, Sze HC, et al. Evolution of treatment for nasopharyngeal cancer-success and setback in the intensity-modulated radiotherapy era. Radiotherapy and Oncology. 2014; 110: 377-84.

15. Hammerlid E, Wirblad B, Sandin C, Mercke C, Edström S, Kaasa S, et al. Malnutrition and food intake in relation to quality of life in head and neck cancer patients. Head \& Neck: Journal for the Sciences and Specialties of the Head and Neck. 1998; 20: 540-8.

16. Turner SL, Tiver K. Synchronous radiotherapy and chemotherapy in the treatment of nasopharyngeal carcinoma. International Journal of Radiation Oncology* Biology* Physics. 1993; 27: 371-7.

17. Li G, Gao J, Liu ZG, Tao YL, Xu BQ, Tu ZW, et al. Influence of pretreatment ideal body weight percentile and albumin on prognosis of nasopharyngeal carcinoma: Long-term outcomes of 512 patients from a single institution. Head \& neck. 2014 ; 36 : 660-6.

18. Nam J-m, McLaughlin JK, Blot WJ. Cigarette smoking, alcohol, and nasopharyngeal carcinoma: a case-control study among US whites. JNCI: Journal of the National Cancer Institute. 1992; 84: 619-22.

19. Bossola M. Nutritional interventions in head and neck cancer patients undergoing chemoradiotherapy: a narrative review. Nutrients. 2015; 7: 265-76.

20. Yilmaz M, Atilla F, Uluer H, Sahin F, Saydam G. Effect of malnutrition on mortality with new espen criteria in patients with hematological malignancies. Clinical Nutrition. 2018; 37: S197-S8.

21. Gautam AP, Fernandes DJ, Vidyasagar MS, Maiya AG, Nigudgi S. Effect of low-level laser therapy on patient reported measures of oral mucositis and quality of life in head and neck cancer patients receiving chemoradiotherapy - a randomized controlled trial. Supportive Care in Cancer. 2013: $21: 1421-8$ 
22. Harada K, Ferdous T, Horinaga D, Uchida K, Mano T, Mishima K, et al. Efficacy of elemental diet on prevention for chemoradiotherapy-induced oral mucositis in patients with oral squamous cell carcinoma. Supportive Care in Cancer. 2016; 24: 953-9.

23. ALLRED CRG, Voss AC, Finn SC, McCAMISH MA. Malnutrition and clinical outcomes: the case for medical nutrition therapy. Journal of the American Dietetic Association. 1996; 96: 361-9.

24. Gillison ML. Current topics in the epidemiology of oral cavity and oropharyngeal cancers. Head \& Neck: Journal for the Sciences and Specialties of the Head and Neck. 2007; 29: 779-92.

25. Gritz ER, Carmack CL, de Moor C, Coscarelli A, Schacherer CW, Meyers EG, et al. First year after head and neck cancer: quality of life. Journal of Clinical Oncology. 1999; 17: 352-

26. Fung K, Lyden TH, Lee J, Urba SG, Worden F, Eisbruch A, et al. Voice and swallowing outcomes of an organ-preservation trial for advanced laryngeal cancer. International Journal of Radiation Oncology* Biology* Physics. 2005; 63: 1395-9.

27. Paccagnella A, Morello M, Da Mosto MC, Baruffi C, Marcon ML, Gava A, et al. Early nutritional intervention improves treatment tolerance and outcomes in head and neck cancer patients undergoing concurrent chemoradiotherapy. Supportive care in cancer. 2010; 18: 837-45.

28. Jin T, Li K-X, Li P-J, Huang S, Chen X-Z, Chen M, et al. An evaluation of nutrition intervention during radiation therapy in patients with locoregionally advanced nasopharyngeal carcinoma. Oncotarget. 2017; 8: 83723.

29. Langius JA, Zandbergen MC, Eerenstein SE, van Tulder MW, Leemans CR, Kramer MH, et al. Effect of nutritional interventions on nutritional status, quality of life and mortality in patients with head and neck cancer receiving (chemo) radiotherapy: a systematic review. Clinical nutrition. 2013; 32: 671-8.

30. Brizel DM, Albers ME, Fisher SR, Scher RL, Richtsmeier WJ, Hars V, et al. Hyperfractionated irradiation with or without concurrent chemotherapy for locally advanced head and neck cancer. New England Journal of Medicine. 1998; 338: 1798-804.

31. Valentini V, Marazzi F, Bossola M, Miccichè F, Nardone L, Balducci M, et al. Nutritional counselling and oral nutritional supplements in head and neck cancer patients undergoing chemoradiotherapy. Journal of human nutrition and dietetics. 2012; 25: 201-8.

32. Williams GF, Teo MT, Sen M, Dyker KE, Coyle C, Prestwich RJ. Enteral feeding outcomes after chemoradiotherapy for oropharynx cancer: a role for a prophylactic gastrostomy? Oral oncology. 2012; 48: 434-40.

33. Arends J, Bachmann P, Baracos V, Barthelemy N, Bertz H, Bozzetti F, et al. ESPEN guidelines on nutrition in cancer patients. Clinical Nutrition. 2017; 36: $11-48$.

34. Loser C, Aschl G, Hebuterne X, Mathus-Vliegen E, Muscaritoli M, Niv Y, et al. ESPEN guidelines on artificial enteral nutrition--percutaneous endoscopic gastrostomy (PEG). Clin Nutr. 2005; 24: 848-61.

35. Magné N, Marcy P, Foa C, Falewee M, Schneider M, Demard F, et al. Comparison between nasogastric tube feeding and percutaneous fluoroscopic gastrostomy in advanced head and neck cancer patients. European archives of oto-rhino-laryngology. 2001; 258: 89-92.

36. Cox JD, Pajak TF, Marcial VA, Coia L, Mohiuddin M, Fu KK, et al. interruptions adversely affect local control and survival with hyperfractionated radiation therapy of carcinomas of the upper respiratory and digestive tracts new evidence for accelerated proliferation from radiation therapy oncology group protocol 8313. Cancer. 1992; 69: 2744-8.

37. Kwong DL, Sham JS, Chua DT, Choy DT, Au GK, Wu P. The effect of interruptions and prolonged treatment time in radiotherapy for nasopharyngeal carcinoma. International Journal of Radiation Oncology* Biology* Physics. 1997; 39: 703-10.

38. Van den Bogaert W, Van der Leest A, Rijnders A, Delaere P, Thames H, van der Schueren E. Does tumor control decrease by prolonging overall treatment time or interrupting treatment in laryngeal cancer? Radiotherapy and Oncology. 1995; 36: 177-82.

39. Piquet M-A, Ozsahin M, Larpin I, Zouhair A, Coti P, Monney M, et al. Early nutritional intervention in oropharyngeal cancer patients undergoing radiotherapy. Supportive care in cancer. 2002; 10: 502-4. 